\title{
Short Communication: Survival and growth of monoculture and mixed-species plantations on the Coromandel coast of India
}

\author{
MUNYSAMY ANBARASHAN", ANBARASHAN PADMAVATHY, RAMADOSS ALEXANDAR \\ Department of Ecology and Environmental Sciences, Pondicherry University. Puducherry, India. `email: anbupu@ gmail.com
}

Manuscript received: 19 November 2017. Revision accepted: 7 December 2017.

\begin{abstract}
Anbarashan M, Padmavathy A, Alexandar R. 2017. Short Communication: Survival and growth of monoculture and mixedspecies plantations on the Coromandel coast of India. Asian J For 1: 70-76. There exists very little information on the growth of autochthonous tree species in the tropics, and when it is compared to monoculture plantations. The aim of this study was to compare the variation in growth parameters between the mixed-species plantation and mono species plantation. The survival and growth in terms of height and girth of 82 autochthonous mixed-species plantations were compared with Casuarina equisetifolia, an exotic species broadly planted in this region after over a decade (2006 to 2016). In the mixed-species plantation, seven species showed 100\% survival rate and 19 species were not survived after 10-year intervals. In the mono species plantation, Casuarina equisetifolia had $92 \%$ survival rate. When it is compared to the monoculture plantation, the growth rate of mixed-species plantation showed highly significant differences $(\mathrm{P}$ $<0: 05)$ values. Simple linear regression between annual girth increment and height produced very strong positive relations $\left(\mathrm{R}^{2} 0.759\right)$. Plantations of Casuarina equisetifolia seem to be well adapted to the coastal region, especially in the Coromandel coast of India. On the other hand, mixed plantations with autochthonous species would contribute more to sustainable management because they provide a greater range of ecological goods and ecosystem services than single-species plantations.
\end{abstract}

Keywords: Exotic species, growth, mortality, autochthonous tree species, plantation, survival

\section{INTRODUCTION}

In tropical countries, there is an increasing interest in establishing mixed autochthonous species plantations for a wide range of economic, silvicultural and sustainability objectives (Nguyen et al. 2016). This is in contrast to the dominance of monoculture plantation at an industrial scale practiced in the sub-tropics and temperate regions, largely because of the association with economic benefits. Mixed plantation systems provide a broader range of options for the restoration of degraded areas, protection and biodiversity conservation (Montagnini et al. 1995; Keenan et al. 1995; Guariguata et al. 1995; Parrotta and Knowles 1999). For example, Vietnam, China, and the Philippines encourage landholders to plant a mixture of tree species in their national reforestation programs (Lamb et al. 2005; Herbohn et al. 2014).

In certain situations, mixed species plantations are found to be more successful in terms of biomass production and carbon sequestration (Lawson and Michler 2014; Puettmann and Tappeiner 2014), improved nutrient cycling (Forrester et al 2010; le Maire et al 2013), reduced damage from pest or disease (Nichols et al 2006; Hung et al 2011), than monocultures. Ecological disturbance and climate change impacts can be mitigated and more resilient forests can be developed when mixtures of different species with different traits are established (Rodrigues et al. 2011). Lamb and Lawrence (1993) stated that the complete utilization of soil and water resources, as well as different soil strata, could be attained by roots of different species during plantation. Plantation of different species tends to observe more solar energy, and the light requirements are broadly distributed in the vertical plane (Guariguata et al. 1995).

The primary goal of ecological research in tropical forests is about comprehending the patterns of highly dynamic plant growth. Forest growth function is important for determining the size and multitude in ecological management and applications (Vivek et al. 2016). Providing practical and meaningful classification of tropical forest species is needed by foresters in modeling the growth and yield factors, whereas the ecologists explain the life history of tropical forests and their diversity (Vivek et al. 2016).

In the prediction of forest dynamics, tree mortality is inevitable, and its center to any long-term dynamics of woody plants as their biomass is regulated by the difference between gains through individual growth and losses through mortality (Scherer-Lorenzen et al. 2005). The growth and mortality of saplings of trees are dependent on impacts of various factors such as species-specific, tree vigor and size, and environmental conditions on the interactions and processes in stands (Scherer-Lorenzen et al. 2005; Radosevich et al. 2006). Differences in mortality rates among species are the major determinants of ecological succession (Schneider et al. 2014) and forest stand structure (Semwal et al. 2013). Performance of a tree species is indicated by its vigor and size, as it partially 
reflects the competitive ability of a tree (Nakashizuka 2001). Growth-mortality trade-off can also be predicted by their relationship to plant functional traits (Baker et al. 2004; Nguyen et al. 2016).

However, the success of the establishment of mixedspecies plantations depends on plantation design and an appropriate definition of the species to be used, taking into consideration ecological and silvicultural aspects (Wormald 1992). There is very little information on the growth of autochthonous tree species in the tropics, and on the comparison between mono and mixed-species plantations. Therefore, this study aimed to determine the growth and survival of 82 autochthonous species in the mixed plantations in the coastal sand dunes compared with Casuarina equisetifolia monoculture plantation after over a decade (2006-2016). The hypotheses tested were: there is variation in growth and survival among species, and the growth and survival of autochthonous species are higher in mixed-species plantations than that in mono-species plantations.

\section{MATERIALS AND METHODS}

\section{Study site}

The study plots were developed in 2006 in Koonimedu Coastal village on the Coromandel Coast of southern India. The mean annual maximum and minimum temperatures are $33^{\circ} \mathrm{C}$ and $24.5^{\circ} \mathrm{C}$. The mean annual rainfall is $1282 \mathrm{~mm}$ year with a six-month dry period (2006 to 2016). In general, coastal sandy soils with poor nutrients prevail in the region.

\section{Preparation of plant materials}

The saplings were produced in the onsite nursery, under a standard white polyethylene nursery bag system. Seeds were collected from the local Tropical Dry Evergreen forests in the region. Weeding became the primary maintenance activity after field planting of trees, and pruning of secondary apical shoots was conducted in the first year.

\section{Research design}

A total of 2055 individuals of 82 autochthonous tree species and 1500 individuals of Casuarina equisetifolia were planted on two hectares in 2006. Table 1 shows the list of species, families, and ecological importance. Species choice was based on growth rate, timber, ecological significance. In each one-hectare plot, diameter at breast height $(\mathrm{dbh})$ and total height were measured for each tree after over a decade (2016). The averages of total height, $\mathrm{dbh}$, basal area, and survival and mortality were calculated for each one-hectare plot in each species. The differences in diameter distribution of trees between the two inventories (2006-2016) were tested using KolmogorovSmirnov two-sample test (Zarr 2006), and we used paired ttests to test the significant differences in tree variables in two different plantations using SPSS software.

Table 1. List of species observed in this study with families and ecological importance

\begin{tabular}{|c|c|c|}
\hline Species & Family & Ecological values \\
\hline \multicolumn{3}{|l|}{ Mixed species } \\
\hline Aegle marmelos (L.) Correa & Rutaceae & Medicinal, economic \\
\hline Aglaia elaeagnoidea (Juss.) Benth. & Meliaceae & Ecological \\
\hline Alangium salvifolium (L.f.) Wangerin & Alangiaceae & Medicinal \\
\hline Albizia amara (Roxb.) Boivin & Mimosaceae & Medicinal, commercial \\
\hline Atalantia monophylla (L.) Correa & Rutaceae & Medicinal, ecological \\
\hline Azadirachta indica A. Juss. & Meliaceae & Medicinal, cultural \\
\hline Barringtonia acutangula (L.) Gaertner & Barringtoniaceae & Ecological \\
\hline Bauhinia purpurea Lam. & Leguminosae & Medicinal \\
\hline Bauhinia racemosa Lam. & Leguminosae & Timber, ecological \\
\hline Benkara malabarica (Lam.) Tirven. & Rubiaceae & Ecological \\
\hline Calophyllum inophyllum $\mathrm{L}$. & Calophyllaceae & Medicinal \\
\hline Calotropis gigantea $\mathrm{L}$. & Apocynaceae & Medicinal, cultural \\
\hline Carmona retusa (Vahl) Masm & Boraginaceae & Ecological \\
\hline Canthium dicoccum (Gaertn.) Merr. & Rubiaceae & Medicinal \\
\hline Cassia auriculata L. & Fabaceae & Medicinal \\
\hline Cassia fistula $\mathrm{L}$. & Fabaceae & Ecological \\
\hline Cassine glauca Rottb. Kuntze. & Celastraceae & Ecological \\
\hline Chloroxylon swietenia DC. & Rutaceae & Timber \\
\hline Coccoloba uvifera $\mathrm{L}$. & Polygonaceae & Fruit, ecological \\
\hline Commiphora berryi (Arn.) Engl. & Burseraceae & Ecological \\
\hline Dalbergia latifolia Roxb. & Fabaceae & Timber \\
\hline Delonix elata Gamble. & Fabaceae & Medicinal, aesthetic \\
\hline Diospyros ebenum J. Koenig ex Retz. & Ebenaceae & Timber \\
\hline
\end{tabular}




\begin{tabular}{|c|c|c|}
\hline Diospyros ferrea (Willd.) Bakh. & Ebenaceae & Ecological \\
\hline Diospyros montana Roxb. & Ebenaceae & Ecological \\
\hline Dolichandrone falcata Seem. & Bignoniaceae & Ecological \\
\hline Drypetes sepiaria (Wight and Arn.) Pax and Hoffm. & Euphorbiaceae & Ecological \\
\hline Ehretia pubescens Benth. & Boraginaceae & Ecological \\
\hline Erythrina indica $\mathrm{L}$. & Fabaceae & Medicinal \\
\hline Eugenia bracteata (Willd.) Roxb. ex DC. & Myrtaceae & Ecological \\
\hline Ficus benghalensis L. & Moraceae & Cultural, medicinal \\
\hline Ficus hispida Lf. & Moraceae & Medicinal \\
\hline Ficus religiosa $\mathrm{L}$. & Moraceae & Cultural, medicinal \\
\hline Garcinia spicata (Wight and Arn.) J.D. Hook. & Clusiaceae & Ecological \\
\hline Glycosmis mauritiana (Lam.) Tanaka & Rutaceae & Fruit, ecological \\
\hline Gliricidia sepium (Jacq.) Kunth ex Walp. & Fabaceae & Medicinal \\
\hline Gmelina asiatica $\mathrm{L}$. & Verbenaceae & Medicinal, aesthetic \\
\hline Helicteres isora $\mathrm{L}$. & Malvaceae & Medicinal, aesthetic \\
\hline Holoptelea integrifolia Planch. & Ulmaceae & Timber \\
\hline Ixora pavetta $\mathrm{T}$. Anderson & Rubiaceae & Cultural, aesthetic \\
\hline Lawsonia inermis $\mathrm{L}$. & Lythraceae & Cultural, medicinal \\
\hline Lepisanthes tetraphylla (Vahl.) Radlk. & Anacardiaceae & Cultural \\
\hline Limonia acidissima $\mathrm{L}$. & Rutaceae & Cultural, medicinal \\
\hline Madhuca longifolia (L.) Macbr. & Sapotaceae & Oil, cultural \\
\hline Maerua oblongifolia Forssk. & Capparaceae & Ecological \\
\hline Mallotus rhamnifolius Muell.-Arg. & Euphorbiaceae & Cultural, aesthetic \\
\hline Manilkara hexandra (Roxb.) Dubard & Sapotaceae & Fruit, ecological \\
\hline Melia azedarach $\mathrm{L}$. & Meliaceae & Medicinal \\
\hline Memecylon umbellatum Burm.f. & Melastomataceae & Ecological, aesthetic \\
\hline Mimusops elengi L. & Sapotaceae & Medicinal, cultural \\
\hline Mitragyna parviflora (Roxb.)Korth. & Rubiaceae & Timber \\
\hline Murraya paniculata $(\mathrm{L})$ Jack & Rutaceae & Aesthetic \\
\hline Ochna obtusata DC. & Ochnaceae & Ecological, aesthetic \\
\hline Ormocarpum sennoides (Willd.)DC. & Leguminosae & Medicinal \\
\hline Pamburus missionis (Wight) Swingle & Rutaceae & Ecological \\
\hline Pandanus oddaratissimus L.f. & Pandanaceae & Ecological \\
\hline Phyllanthus reticulatus Poir. & Euphorbiaceae & Ecological, medicinal \\
\hline Pleiospermium alatum (Wall. ex Wight. \& Arn.) Swingle & Rutaceae & Ecological, medicinal \\
\hline Polyalthia suberosa (Dunal) Thw. & Annonaceae & Ecological, aesthetic \\
\hline Pongamia pinnata $($ L.) Pierre & Fabaceae & Oil, cultural \\
\hline Pterocarpus marsupium Roxb. & Combretaceae & Timber, medicinal \\
\hline Pterospermum canescens Roxb. & Sterculiaceae & Ecological \\
\hline Pterospermum xylocarpum (Gaertn.) Sant. \& Wagh. & Sterculiaceae & Ecological \\
\hline Salacia chinensis $\mathrm{L}$. & Celastraceae & Medicinal \\
\hline Salvadora persica $\mathrm{L}$. & Salvadoraceae & Medicinal, economic \\
\hline Sapindus emarginatus Vahl & Sapindaceae & Medicinal, economic \\
\hline Streblus asper Lour. & Moraceae & Ecological \\
\hline Strychnos nux-vomica $\mathrm{L}$. & Loganiaceae & Medicinal \\
\hline Strychnos potatorum Lf. & Loganiaceae & Ecological, medicinal \\
\hline Suregada angustifolia ( Baill. ex. Muell-Arg.) Airy Shaw & Meliaceae & Ecological \\
\hline Syzygium cumini (L.) Skeels & Myrtaceae & Medicinal, fruit \\
\hline Tarenna asiatica (L.) Kuntze. & Rubiaceae & Medicinal, cultural \\
\hline Terminalia arjuna (DC.) Wight \& Arn. & Combretaceae & Medicinal, timber \\
\hline Terminalia bellirica (Gaertner) Roxb. & Combretaceae & Medicinal, timber \\
\hline Terminalia catappa $\mathrm{L}$. & Combretaceae & Ecological, fruit \\
\hline Thespesia populnea (L.)Sol. & Malvaceae & Timber, cultural \\
\hline Tricalysia sphaerocarpa (Dalz.) Gamble & Rubiaceae & Timber, cultural \\
\hline Vitex leucoxylon Lf. & Lamiaceae & Timber, ecological \\
\hline Vitex negundo L. & Lamiaceae & Medicinal, cultural \\
\hline Walsura trifolia (A..Juss.) Harms & Rubiaceae & Ecological \\
\hline Wrightia tinctoria (Roxb.) R.Br. & Apocynaceae & Medicinal \\
\hline Ziziphus mauritina Lam. & Rhamnaceae & Ecological \\
\hline \multicolumn{3}{|l|}{ Monoculture plantation } \\
\hline Casuarina equisetifolia $\mathrm{L}$. & Casuarinaceae & Fuel wood \\
\hline
\end{tabular}




\section{RESULTS AND DISCUSSION}

Measurements in the mixed-species plantation at 10 years of age showed that Albizia amara, Lepisanthes tetraphylla, Diospyros ferrea, Eugenia bracteata, Mimusops elengi, Sapindus emarginata, and Terminalia bellerica exhibited the highest rate of survival (100\%), followed by Wrightia tinctoria, Mitragyna parviflora, Streblus asper, Pleiospermium alatum, Gmelina asiatica, Ixora pavetta and Coccoloba uvifera showing $99 \%$ of the survival rate (Table 2). In total, 19 species were not survived for over a decade. No species exhibited significant differences $(\mathrm{P}<0.05)$ in survival between the mixed species and monoculture plantation plots. Species such as Bauhinia purpurea, Benkara malabarica, Calophyllum inophyllum, Limonia acidissima, Polyalthia suberosa, Pterospermum xylocarpum, Strychnos potatorum, Terminalia catappa and Thespesia populnea did not survive any single sapling in the two-hectare plots. Barringtonia acutangula, Cassia fistula, Chloroxylon swietenia, Pamburus missionis and Pterocarpus marsupium demonstrated less than $20 \%$ survival rates. Comparing monoculture to mixed-species plantations, in general, species in the monoculture plantation demonstrated better survival rates. Notably, in the single species plot, Casuarina equisetifolia exhibited high survival and growth rates. Introducing new species, however, is not without risks. Many reforestation projects fail due to inappropriate species choice, a consequence of inadequate knowledge about the potential of species and their growth and survival rates under different site and environmental conditions (Corlett 1999; Wuethrich 2007; Rodrigues et al. 2009).

The use of a wider variety of autochthonous species in reforestation may enhance the recovery of ecosystems, decrease sensitivity to pests and diseases, and increase functional diversity (Hooper et al. 2005; Benayas et al. 2009; Rodrigues et al. 2009). Creation of forests in the tropics takes place across a wide variety of non-climatic and climatic conditions. Different reforestation experiments have elucidated that environmental conditions may have strong effects on species growth and survival (Butterfield 1996; Calvo-Alvarado et al. 2007; Park et al. 2010). On the other hand, the finding that $23 \%$ of the species may have high initial mortality and unsatisfactory early growth is critical information in avoiding early failure of reforestation projects. Several species showed poor performance and seemed to be unsuitable for large-scale planting in open plantation sites. Ashton et al. (2001) reported that some of these species might do better when they were planted later after some pioneer plants or extant nurse trees.

In the mixed-species plantation, the measurements taken at 10 years intervals showed that Ficus benghalensis and Bauhinia racemosa have the best growth in terms of height, followed by A. amara and Azadirachta indica, with no statistically significant differences $(\mathrm{P}<0.05)$ between monoculture and mixed autochthonous species plantations. In the monoculture plantation, $C$. equisetifolia showed moderate growth of height and girth. Simple linear regression between annual girth increment and height produced very strong positive relation $\left(\mathrm{R}^{2} 0.759\right)$ (Figure 1).

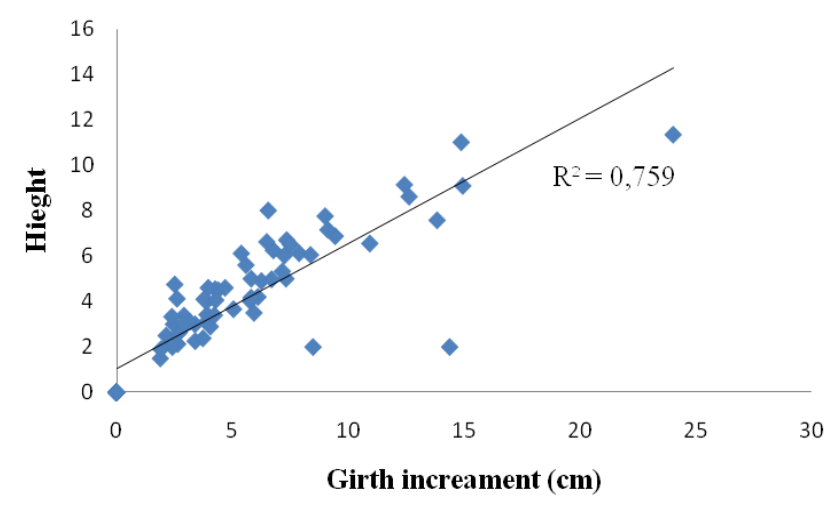

Figure 1. Simple linear regression between annual girth increment and average height of mixed-species plantation (2 ha).

Table 2. Number of individuals of each species with survival and growth rate after 10 year period of intervals

\begin{tabular}{|c|c|c|c|}
\hline Species & $\begin{array}{c}\text { Planted in } \\
2006\end{array}$ & $\begin{array}{c}\text { Survived in } \\
2016\end{array}$ & $\begin{array}{c}\text { Mean annual } \\
\text { girth increment }(\mathrm{cm})\end{array}$ \\
\hline \multicolumn{4}{|l|}{ Mono plantation } \\
\hline Casuarina equisetifolia $\mathrm{L}$. & 1500 & 1380 & $14.564 \pm 0.478$ \\
\hline \multicolumn{4}{|l|}{ Mixed species } \\
\hline Aegle marmelos (L.) Correa & 10 & 8 & $2.337 \pm 0.678$ \\
\hline Aglaia elaeagnoidea (Juss.) Benth. & 4 & 4 & $2.774 \pm 0.478$ \\
\hline Alangium salvifolium (L.f.) Wangerin & 26 & 22 & $2.945 \pm 1.317$ \\
\hline Albizia amara (Roxb.) Boivin & 40 & 40 & $14.978 \pm 9.127$ \\
\hline Atalantia monophylla (L.) Correa & 50 & 31 & $2.464 \pm 0.863$ \\
\hline Azadirachta indica A. Juss. & 20 & 18 & $12.65 \pm 4.608$ \\
\hline Barringtonia acutangula (L.) Gaertner & 10 & 1 & 14.4 \\
\hline Bauhinia purpurea Lam. & 25 & 0 & 0 \\
\hline Bauhinia racemosa Lam. & 150 & 145 & $12.458 \pm 5.055$ \\
\hline Benkara malabarica (Lam.) Tirven. & 20 & 0 & 0 \\
\hline Calophyllum inophyllum L. & 15 & 0 & 0 \\
\hline Calotropis gigantea $\mathrm{L}$. & 10 & 4 & $2.525 \pm 0.853$ \\
\hline Carmona retusa (Vahl) Masm & 35 & 29 & $2.658 \pm 0.797$ \\
\hline
\end{tabular}




\begin{tabular}{|c|c|c|c|}
\hline Canthium dicoccum (Gaertn.) Merr. & 10 & 10 & $3.95 \pm 2.204$ \\
\hline Cassia auriculata $\mathrm{L}$. & 20 & 13 & $7.36 \pm 3.509$ \\
\hline Cassia fistula $\mathrm{L}$. & 10 & 2 & $1.9 \pm 0.707$ \\
\hline Cassine glauca Rottb. Kuntze. & 30 & 28 & $6.275 \pm 3.750$ \\
\hline Chloroxylon swietenia DC. & 10 & 2 & $4.4 \pm 1.414$ \\
\hline Coccoloba uvifera $\mathrm{L}$. & 30 & 29 & $5.786 \pm 4.142$ \\
\hline Commiphora berryi (Arn.) Engl. & 100 & 81 & $7.907 \pm 3.142$ \\
\hline Dalbergia latifolia Roxb. & 5 & 4 & $5.4 \pm 1.914$ \\
\hline Delonix elata Gamble. & 15 & 12 & $5.608 \pm 3.538$ \\
\hline Diospyros ebenum J. Koenig ex Retz. & 70 & 69 & $4.066 \pm 2.681$ \\
\hline Diospyros ferrea (Willd.) Bakh. & 70 & 70 & $4.271 \pm 2.534$ \\
\hline Diospyros montana Roxb. & 20 & 18 & $2.927 \pm 1.143$ \\
\hline Dolichandrone falcata Seem. & 50 & 45 & $6.122 \pm 4.170$ \\
\hline Drypetes sepiaria (Wight and Arn.) Pax and Hoffm. & 28 & 26 & $3.419 \pm 1.808$ \\
\hline Ehretia pubescens Benth. & 10 & 0 & 0 \\
\hline Erythrina indica $\mathrm{L}$. & 10 & 0 & 0 \\
\hline Eugenia bracteata (Willd.) Roxb. ex DC. & 20 & 20 & $2.425 \pm 2.009$ \\
\hline Ficus benghalensis L. & 5 & 3 & $24.066 \pm 9.928$ \\
\hline Ficus hispida Lf. & 10 & 0 & 0 \\
\hline Ficus religiosa L. & 1 & 1 & 14.9 \\
\hline Garcinia spicata (Wight and Arn.) J.D. Hook. & 15 & 13 & $3.746 \pm 1.983$ \\
\hline Glycosmis mauritiana (Lam.) Tanaka & 20 & 16 & $1.931 \pm 0.618$ \\
\hline Gliricidia sepium (Jacq.) Kunth ex Walp. & 5 & 0 & 0 \\
\hline Gmelina asiatica $\mathrm{L}$. & 25 & 24 & $6.796 \pm 3.175$ \\
\hline Helicteres isora $\mathrm{L}$. & 30 & 28 & $3.978 \pm 2.404$ \\
\hline Holoptelea integrifolia Planch. & 90 & 82 & $7.332 \pm 4.175$ \\
\hline Ixora pavetta $\mathrm{T}$. Anderson & 20 & 19 & $3.924 \pm 1.219$ \\
\hline Lawsonia inermis L. & 5 & 4 & $3.9 \pm 1.732$ \\
\hline Lepisanthes tetraphylla (Vahl.) Radlk. & 101 & 101 & $7.172 \pm 4.037$ \\
\hline Limonia acidissima L. & 5 & 0 & 0 \\
\hline Madhuca longofolia (L.) Macbr. & 5 & 3 & $5.066 \pm 4.618$ \\
\hline Maerua oblongifolia Forssk. & 5 & 0 & 0 \\
\hline Mallotus rhamnifolius Muell.-Arg. & 5 & 0 & 0 \\
\hline Manilkara hexandra (Roxb.) Dubard & 85 & 83 & $6.719 \pm 3.075$ \\
\hline Melia azedarach L. & 5 & 3 & $6.566 \pm 5.107$ \\
\hline Memecylon umbellatum Burm.f. & 5 & 2 & $2.15 \pm 0.535$ \\
\hline Mimusops elengi $\mathrm{L}$. & 35 & 35 & $5.82 \pm 3.083$ \\
\hline Mitragyna parviflora (Roxb.)Korth. & 15 & 15 & $4.233 \pm 2.135$ \\
\hline Murraya paniculata (L) Jack & 10 & 7 & $2.471 \pm 0.449$ \\
\hline Ochna obtusata DC. & 10 & 7 & $7.525 \pm 3.224$ \\
\hline Ormocarpum sennoides (Willd.)DC. & 10 & 1 & 3.4 \\
\hline Pamburus missionis (Wight) Swingle & 5 & 0 & 0 \\
\hline Pandanus oddaratissimus L.f. & 10 & 9 & $3.177 \pm 0.440$ \\
\hline Phyllanthus reticulatus Poir. & 20 & 0 & 0 \\
\hline Pleiospermium alatum (Wall. ex Wight. \& Arn.) Swingle & 100 & 88 & $8.396 \pm 5.134$ \\
\hline Polyalthia suberosa (Dunal) Thw. & 5 & 0 & 0 \\
\hline Pongamia pinnata $($ L.) Pierre & 5 & 0 & 0 \\
\hline Pterocarpus marsupium Roxb. & 5 & 1 & 8.5 \\
\hline Pterospermum canescens Roxb. & 50 & 42 & $7.269 \pm 4.281$ \\
\hline Pterospermum xylocarpum (Gaertn.) Sant. \& Wagh. & 10 & 0 & 0 \\
\hline Salacia chinensis $\mathrm{L}$. & 5 & 5 & $4.7 \pm 3.383$ \\
\hline Salvadora persica L. & 20 & 16 & $2.622 \pm 1.617$ \\
\hline Sapindus emarginatus Vahl & 40 & 40 & $6.5 \pm 4.071$ \\
\hline Streblus asper Lour. & 30 & 29 & $3.796 \pm 2.114$ \\
\hline Strychnos nux-vomica L. & 35 & 31 & $2.722 \pm 1.235$ \\
\hline Strychnos potatorum Lf. & 10 & 0 & 0 \\
\hline Suregada angustifolia ( Baill. ex. Muell-Arg.) Airy Shaw & 10 & 8 & $5.837 \pm 3.580$ \\
\hline Syzygium cumini (L.) Skeels & 10 & 8 & $9.462 \pm 4.617$ \\
\hline Tarenna asiatica (L.) Kuntze. & 5 & 2 & $2.9 \pm 1.414$ \\
\hline Terminalia arjuna (DC.) Wight \& Arn. & 10 & 9 & $10.955 \pm 4.126$ \\
\hline Terminalia bellirica (Gaertner) Roxb. & 10 & 10 & $5.95 \pm 4.126$ \\
\hline Terminalia catappa $\mathrm{L}$. & 30 & 0 & 0 \\
\hline Thespesia populnea (L.) Sol. & 25 & 0 & 0 \\
\hline Tricalysia sphaerocarpa (Dalz.) Gamble & 5 & 3 & $2.4 \pm 0.866$ \\
\hline Vitex leucoxylon Lf. & 15 & 14 & $13.864 \pm 5.607$ \\
\hline Vitex negundo L. & 10 & 8 & $9.025 \pm 2.100$ \\
\hline Walsura trifolia (A..Juss.) Harms & 50 & 48 & $4.29 \pm 4.16$ \\
\hline Wrightia tinctoria (Roxb.) R.Br. & 70 & 69 & $9.146 \pm 3.860$ \\
\hline Ziziphus mauritina Lam. & 10 & 0 & 0 \\
\hline Total & 2055 & 1616 & \\
\hline
\end{tabular}


The growth in diameter of Ficus benghalensis was the highest in the mixed autochthonous species plantation plot, followed by A. amara, Vitex leucoxylon, and A. indica with no statistically significant differences $(\mathrm{P}<0.05)$ with that in the mixed-species plots. When compared to the monoculture plantation, it showed highly significant differences $(\mathrm{P}<0.05)$ values. In the monoculture plantation, Casuarina equisetifolia showed a greater diameter increment in the last 10 years when compared to the mixed-species plantation. Tricalysia sphaerocarpa, Tarenna asiatica, Strychnos nux-vomica, Salvadora persica, Murraya paniculata, Glycosmis mauritiana, Cassia fistula, and Aegle marmelos showed the slowest growth rates, with no significant differences in the mixed plantation. Casuarina equisetifolia had the highest basal area, showing significant differences $(\mathrm{P}<0.05)$ compared to all species and the mixture of autochthonous species plantations. The present study revealed that the variation in GBH increment was also found on trees from similar species. This might be due to the response of each species to the growth process, which was different among species as well as among trees of similar species. Many studies showed that the internal and external factors had affected tree growth and development (Breugel et al. 2011). The internal factors comprised genetic factors, plant growth process, internal growth property, and physiological process. On the other hand, the soil parameters, microclimatic factors, and response plants to the environment could be the external factors. Miya et al. (2009) reported that variation in diameter growth of different saplings of different species in an uneven-aged mixed stand was influenced by individual growth conditions, but it was negatively related to the wood density (Keeling et al. 2008).

In conclusion, the present study shows that both monoculture and mixed autochthonous species can perform well in the plantation sites. Although the plantations are still young and it may be too soon to determine the behavior of the species studied, there is evidence that the best growth for these species was demonstrated in mixed autochthonous species systems. The higher mortality of shade-intolerant species appears to be the result due to the high intensity of light in coastal dune ecosystem. Management practices such as pruning and thinning could favor the development of these species in mixed plantations and provide revenues at earlier ages when an appropriate group of species is used. Plantations of Casuarina equisetifolia seem to be well adapted to the coastal region and are certainly commercially important trees. On the other hand, mixed species plantations with autochthonous species would contribute more to sustainable management because they provide a greater range of ecological goods and ecosystem services than monoculture plantations.

\section{ACKNOWLEDGEMENTS}

The financial assistance from Pitchandikulam Forest, Auroville (PFs), India is gratefully acknowledged. We really express our gratitude to the Tamil Nadu Forest Department for permitting us to conduct the field study.

\section{REFERENCES}

Ashton PMS, Gunatilleke CVS, Singhakumara BMP, Gunatilleke IAUN. 2001. Restoration pathways for rain forest in southwest Sri Lanka: a review of concepts and models. For Ecol Manag 154: 409-430. DOI: 10.1016/S0378-1127(01)00512-6.

Baker TR, Phillips OL, Malhi Y, Almeida S, Arroyo L, Di Fiore A. 2004. Variation in wood density determines spatial patterns in Amazonian forest biomass. Global Ch Biol 10 (5): 545-62. DOI: 10.1111/j.13652486.2004.00751.x

Benayas JMR, Newton AC, Diaz A., Bullock JM. 2009. Enhancement of biodiversity and ecosystem services by ecological restoration: a metaanalysis. Science 325: 1121-1124. DOI: 10.1126/science.1172460.

Butterfield R. 1996. Early species selection for tropical reforestation: a consideration of stability. For Ecol Manag 81: 161-168. DOI: 10.1016/0378-1127(95)03649-0.

Calvo-Alvarado JC, Arias D, Richter DD. 2007. Early growth performance of autochthonous and introduced fast-growing tree species in wet to sub-humid climates of the Southern region of Costa Rica. For Ecol Manag 242: 227-235. DOI: 10.1016/j.foreco.2007.01.034.

Corlett RT. 1999. Environmental forestry in Hong Kong: 1871-1997. For Ecol Manag 116: 93-105. DOI: 10.1016/S0378-1127(98)00443-5.

Forrester DI, Theiveyanathan S, Collopy JJ, Marcar NE. 2010. Enhanced water use efficiency in a mixed Eucalyptus globulus and Acacia mearnsii plantation. For Ecol Manag 259 (9): 1761-1770. DOI: 10.1016/j.foreco.2009.07.036.

Guariguata MR, Rheingans R, Montagnini F. 1995. Early woody invasion under tree plantations in Costa Rica: implications for forest restoration. Restor Ecol 3: 252-260. DOI: 10.1111/j.1526100X.1995.tb00092.x

Herbohn JL, Vanclay J, Nguyen H, Le HD, Baynes JR, Harrison S, Cedamon E, Smith C, Firn J, Gregorio NO, Mangaoang E, Lamarre E. 2104. Inventory Procedures for Smallholder and Community Woodlots in the Philippines: Methods, Initial Findings and Insights. Small-scale For 13: 79-100. DOI: 10.1007/s11842-013-9242-6.

Hooper DU, Chapin FS, Ewel JJ, Hector A, Inchausti P, Lavorel S, Lawton JH, Lodge DM, Loreau M, Naeem S, Schmid B, Setälä H, Symstad AJ, Vandermeer J, Wardle DA. 2005. Effects of biodiversity on ecosystem functioning: a consensus of current knowledge. Ecol Monogr 75: 3-35. DOI: 10.1890/04-0922.

Hung TD, Herbohn JL, Lamb D, Nhan HD. 2011. Growth and production varies between pair-wise mixtures and monoculture plantations in North Viet Nam. For Ecol Manag 262 (3): 440-448. DOI: 10.1016/j.foreco.2011.04.010.

Keeling HC, Baker TR, Martinez RV, Monteagudo A, Phillips OL. 2008. Contrasting patterns of diameter and biomass increment across tree functional groups in Amazonian forests. Oecologia 158: 521-534. Doi: 10.1007/s00442-008-1161-4.

Lamb D, Erskine PD, Parrotta JA. 2005. Restoration of degraded tropical forest landscapes. Science. 310 (5754): 1628. DOI: 10.1126/science. 1111773 .

Lamb D, Lawrence P. 1993. Mixed species plantations using high-value rainforest trees in Australia. In: Lieth, H., Lohmann, M. (Eds.), Restoration of Tropical Forest Ecosystems. Kluwer Academic Publishers, Nederland.

Lawson SS, Michler CH. 2014. Afforestation, restoration and regeneration - Not all trees are created equal. J For Res 25 (1): 3-20. DOI: 10.1007/s11676-014-0426-5.

Le Maire G, Nouvellon Y, Christina M, Ponzoni FJ, GoncËalves JLM, Bouillet JP. 2013. Tree and stand light use efficiencies over a full rotation of single-and mixed-species Eucalyptus grandis and Acacia mangium plantations. For Ecol Manag 288: 31-42. DOI: 10.1016/j.foreco.2012.03.005

Breugel MV, Hall JS, Craven DJ, Gregoire TG, Park A, Dent DH, Wishnie MH, Mariscal E, Deago J, Ibarra D, Cedeno N, Ashton MS. 2011. Early growth and survival of 49 tropical tree species across sites differing in soil fertility and rainfall in Panama. For Ecol Manag 261: 1580-1589. DOI: 10.1016/j.foreco.2010.08.019. 
Miya H, Yoshida T, Noguchi M, Nakamura F. 2009. Individual growing conditions that affect diameter increment of tree saplings after selection harvesting in a mixed forest in Northern Japan. J For Res 14: 302-310. DOI: 10.1007/s10310-009-0136-6.

Montagnini F, Gonzalez E, Rheingans R, Porras C. 1995. Mixed and pure forest plantations in the humid neotropics: a comparison of early growth, pest damage and establishment costs. Commonwealth For Rev 74 (4): 306-314

Nakashizuka T. 2001. Species coexistence in temperate, mixed deciduous forests. Trends Ecol Evol 16 (4): 205-210. DOI: 10.1016/S0169 5347(01)02117-6.

Nguyen H, Vanclay J, Herbohn J, Firn N. 2016. Drivers of Tree Growth, Mortality and Harvest Preferences in Species-Rich Plantations for Smallholders and Communities in the tropics. PLoS One. 11 (10) e0164957. DOI: 10.1371/journal.pone.0164957.

Nichols JD, Bristow M, Vanclay JK. 2006. Mixed-species plantations: Prospects and challenges. For Ecol Manag 233 (23): 383-390. DOI 10.1016/j.foreco.2006.07.018.

Park A, van Breugel M, Ashton, PMS, Mariscal E, Deago J, Ibarra D, Cede no N, Hall JS. 2010. Local and regional environmental variation influences the growth of tropical trees in selection trials in the Republic of Panama. For Ecol Manag 260: 12-21. DOI 10.1016/j.foreco.2010.03.021.

Parrotta JA, Knowles OH. 1999. Restoration of Tropical Moist Forests on Bauxite-Mined Lands in the Brazilian Amazon. Restor Ecol 7 (2) 103-116. DOI: 10.1046/j.1526-100X.1999.72001.x

Puettmann KJ, Tappeiner JC. 2014. Multi-scale assessments highligh silvicultural opportunities to increase species diversity and spatia variability in forests. Forestry. 87 (1): 1-10. DOI 10.1093/forestry/cpt050
Radosevich SR, Hibbs DE, Ghersa CM. 2006. Effects of species mixtures on growth and stand development of Douglas-fir and red alder. Canadian J For Res 36 (3): 768-782. DOI: 10.1139/x05-280.

Rodrigues J, de Castro M, Cancho VG, Balakrishnan N. 2009. COMPoisson cure rate survival models and an application to a cutaneous melanoma data. J Statistic Plann Infer 139 (10): 3605-3611. DOI: 10.1016/j.jspi.2009.04.014.

Rodrigues RR, Gandolfi S, Nave AG, Aronson J, Barreto TE, Vidal CY. 2011. Large-scale ecological restoration of high-diversity tropical forests in SE Brazil. For Ecol Manag 261 (10): 1605-1613. DOI: 10.1016/j.foreco.2010.07.005

Scherer-Lorenzen M, Potvin C, Koricheva J, Schmid B, Hector A, Bornik Z. 2005. The Design of Experimental Tree Plantations for Functional Biodiversity Research. In: Scherer-Lorenzen M, KoÈrner C, Schulze E-D, editors. Forest Diversity and Function. Ecol Studies. Springer, Berlin.

Schneider T, Ashton M, Montagnini F, Milan P. 2014. Growth performance of sixty tree species in smallholder reforestation trials on Leyte, Philippines. New Forests 45 (1): 83-96. Doi: 10.1007/s11056013-9393-5.

Semwal RL, Nautiyal S, Maikhuri RK, Rao KS, Saxena KG. 2013. Growth and carbon stocks of multipurpose tree species plantations in degraded lands in Central Himalaya, India. For Ecol Manag 310: 450459. DOI: 10.1016/j.foreco.2013.08.023.

Vivek P, Parthasarathy N, Monica P. 2016. Short-term girth increment in tree species of Tropical Dry Evergreen forest on the Coromandel Coast of India. J Global Ecol Environ 4 (3): 147-152.

Wormald TJ. 1992. Mixed and Pure Forest Plantations in the Tropics and Subtropics, no. 103. FAO, Rome.

Wuethrich B. 2007. Biodiversity: reconstructing Brazil's Atlantic rainforest. Science 315: 1070-1072. DOI: 10.1126/science.315.5815.1070 